\title{
Correction to: The Change of Oxidative Stress in Maternal Blood During Pregnancy
}

\author{
Mika Fukase $^{1}{ }^{1} \cdot$ Norikazu Watanabe $^{1} \cdot$ Keiko Yamanouchi $^{1} \cdot$ Seiji $^{\text {Tsutsumi }}{ }^{1} \cdot$ Satoru Nagase $^{1}$
}

Published online: 2 February 2022

(c) Society for Reproductive Investigation 2022

Correction to: Reprod. Sci.

https://doi.org/10.1007/s43032-022-00848-8

This article was updated to correct the author names, which were transposed.

The original article can be found online at https://doi.org/10.1007/ s43032-022-00848-8.

Mika Fukase

m.fukase3@gmail.com

1 Department of Obstetrics and Gynecology, Faculty

of Medicine, Yamagata University, Iida-nishi, 2-2-2,

Yamagata 990-9585, Japan 